\title{
Worse prognosis of cocaine users with ACS - a call for routine urine tests?
}

Cocaine consumption is associated with more complications and a 10-fold higher in-hospital mortality among patients with acute coronary syndrome (ACS), report Spanish investigators. "The findings were astonishing due to their magnitude," says lead researcher Antoni Bayes-Genis.

The highest level of cocaine consumption in Europe is reported in Spain, where $8.3 \%$ of the adult population have taken the drug. Although cocaine causes known cardiovascular complications, its effect on in-hospital evolution of ACS was unknown.

The investigators included questions about cocaine use in a questionnaire to 479 patients aged $\leq 50$ years admitted to hospital with ACS from 2001 to 2008. Urine tests for cocaine were performed in 403 patients. The prevalence of cocaine use tripled in this population during the study period, reaching $21.7 \%$ in 2008 .

Acute cocaine users ( $4-5 \%$ of patients), as defined by medical history or a positive urine test, had larger infarct areas, lower left ventricular ejection fraction, more-frequent tachycardia and more-severe heart failure than nonacute users or nonusers, which resulted in a substantially worse prognosis. Although limited in size and to a single hospital, the researchers hope their study increases awareness of the harmful consequences of cocaine consumption. In addition, they suggest that routine urine tests in all patients with ACS $\leq 50$ years could help identify cocaine users and treat them more effectively. Bayes-Genis also calls for $\beta$-blockers to be reconsidered for treatment of cocaine users with ACS, because of their useful antiarrhythmic and anti-ischemic properties.

Christoph A. Schmitt

Original article Carrillo, X. et al. Acute coronary syndrome and cocaine use: 8-year prevalence and inhospital outcomes. Eur. Heart J. doi:10.1093/ eurheart/ehq504 